\title{
CARACTERIZACIÓN DE JÓVENES PRIMERA GENERACIÓN EN EDUCACIÓN SUPERIOR. NUEVAS TRAYECTORIAS HACIA LA EQUIDAD EDUCATIVA
}

\section{Introducción}

Los análisis realizados respecto a la equidad del sistema educativo chileno han evidenciado los avances que en esta materia se han logrado en las últimas décadas (García Huidobro y Bellei 2003, García Huidobro 2005 y Navarro 2002). A la cobertura prácticamente universal en enseñanza básica hace ya más de dos décadas, se suma la alta cobertura registrada en los últimos diez años en la educación media $^{2}$, que termina consolidándose en 2003 con la ley que define como educación obligatoria los doce años de escolaridad. Como contracara de estos avances, estos mismos trabajos señalan la dificultad existente para que este acceso cada vez más equitativo se transforme también en resultados educativos de calidad para todos.

Siguiendo esta línea argumentativa, Bellei y García Huidobro (2003) señalan que un indicador que se puede usar para medir el grado de equidad del sistema educativo chileno es la probabilidad que tiene un segmento social en acceder a la educación superior y si esta probabilidad se distribuye equitativamente en todos los segmentos sociales de nuestra población. La evidencia a este respecto es poco feliz si analizamos la cobertura de educación terciaria estimada a partir de la tasa de asistencia neta (Castillo, 2009). En él es posible

2 Es posible estimar la cobertura educativa de un nivel a través de la "Tasa Neta de Asistencia" que se refiere al número total de alumnos en edad de estar cursando el nivel correspondiente y que realmente lo están haciendo, por sobre el total de población de ese mismo rango etario. Generalmente esta información se podría decir que se encuentra subdimensionada ya que no incluye a aquellos niños o jóvenes que están cursando el nivel educativo respectivo, pero que no se encuentran en el tramo etario comúnmente establecido para ese nivel (por ejemplo estudiantes repitentes o adelantados). En el caso de la enseñanza básica podemos ver que la tasa neta de asistencia fluctuó de 90,4\% el año 1990 a 92,4 el año 2006. Para el caso de la enseñanza media la fluctuación es mayor ya que el año 1990 esta era de 60,1\% y el año 2006 llegaba a 71,6\% (Casen 2006). 
observar que la probabilidad de estudios postsecundarios ha mejorado a partir de los años noventa en todos los segmentos sociales, llegando casi a triplicarse para el caso de los dos quintiles más pobres de la población (de un 4\% de probabilidades el año 1990 a un 13,7\% para el año 2006 en el caso del quintil más pobre). Lamentablemente, a pesar de esa importante mejora, hay una brecha significativa entre el segmento más rico y el más pobre a este respecto, llegando a casi un $40 \%$ de diferencia.

En los últimos años, en nuestro país, se ha ido consolidando una visión que muestra una progresiva apertura de la educación superior a segmentos de la población que antiguamente se encontraban excluidos de este nivel educativo. El aumento sostenido en los recursos provistos por el Estado en materia de ayudas estudiantiles para favorecer un acceso más equitativo a este tipo de educación ${ }^{3}$ y el consecuente aumento sostenido de las expectativas de los jóvenes y su entorno familiar por el ingreso a la educación terciaria son muestra de este nuevo escenario ${ }^{4}$.

Esta mayor demanda se evidencia también con el incremento del número de jóvenes que rinden la Prueba de Selección Universitaria, PSU. Por ejemplo, el año 2004 el 61,4\% de los estudiantes de cuarto año medio la rindieron, subiendo a 70,1\% el porcentaje el año 2006 (Castillo, 2007), y llegando al 86,5\% para el proceso de admisión 2009.

Sin duda, la idea que antiguamente hacía referencia a que la educación superior estaba reservada casi exclusivamente para la elite o para aquellos jóvenes meritorios de estratos medios o bajos ha ido

3 Los últimos esfuerzos estatales en educación superior han apuntado a la generación de condiciones de permanencia y acceso de los estudiantes de los quintiles más pobres; así se han duplicado las ayudas estudiantiles para pago de aranceles, y específicamente en los últimos dos años las becas y subsidios se han llegado a triplicar según datos internos de la División de Educación Superior del Ministerio de Educación.

4 Según encuesta a actores educativos de UAH-CIDE 2006, el 83,9\% de los estudiantes secundarios tienen expectativas de acceder a estudios superiores., $10 \%$ más que lo que manifestaban el año 2004 según esta misma encuesta. Este aumento de expectativas ha aumentado transversalmente. Según dependencia de los establecimientos educativos, el año 2006 el 79,6\% de los estudiantes de liceos municipales, el 86,1\% de los que estudian en liceos particulares subvencionados y el $89 \%$ de los particulares pagados tienen expectativas de acceder a la educación superior. 
mutando en los últimos años. Las tasas de cobertura del sistema se han duplicado desde 1992 al 2006, pasando de un 16,3\% a un 34\%. Otra forma de analizar este crecimiento es a través de variaciones de la matrícula, donde esta creció en un 238\% en el periodo 1990-2004 (PNUD, 2005).

Esta creciente apertura educativa se ha ido encarnando en un "sujeto tipo", aquel joven que teniendo padres que no lograron acceder a la formación terciaria logra ingresar a ésta. Ellos se han denominado como "primera generación en la educación superior". Según Armanet (2005) "siete de cada diez jóvenes que estudian una carrera provienen de familias en las cuales ninguno de sus miembros tuvo esa oportunidad". Esto refuerza la idea de una tendencia en expansión en nuestro sistema educativo. Datos más recientes (Meneses, Rolando, Valenzuela y Vega, 2010) señalan que de los alumnos en educación superior, un $80 \%$ pertenece a este grupo que ha logrado superar la educación de sus padres.

Sólo recientemente en nuestro país se estableció un interés por profundizar el conocimiento de este grupo estudiantil. De las cada vez más recurrentes cuantificaciones de este grupo en los últimos cinco años, hemos pasado lentamente al surgimiento de análisis exploratorios que intentan describirlos mejor ${ }^{5}$. Bajo este mismo objetivo, este artículo presenta una nueva aproximación a este fenómeno, por una parte intentando abordarlo desde una perspectiva conceptual, que permita circunscribir el fenómeno en una discusión teórica, y por la otra, abarcándolo metodológicamente de manera longitudinal, más amplia e integral.

En la primera parte del artículo se discuten conceptos como el de "trayectoria", "estrategia" y "movilidad social" que permiten explicar y circunscribir mejor la situación de estos "nuevos estudiantes". En un segundo apartado se realiza una descripción de los "estudiantes primera generación" a partir del contexto sociocultural en que se encuentran, indagando en el capital cultural de sus familias, para luego describir su vida escolar en términos de sus trayectorias y desempeños.

5 Ver CPCE 2009 y Concha, 2009. 
En ambos tipos de análisis se establece como grupo de comparación aquellos jóvenes que también teniendo padres con menos de doce años de escolaridad, no lograron acceder a la educación superior.

En una tercera parte se describe, por un lado, el tipo de acceso a la educación terciaria que estos estudiantes tienen, haciendo mención de las condiciones que lo hicieron posible, del tipo de enseñanza que cursan y, en parte, del desempeño que logran tener en ésta. Considerando que este análisis profundiza en su relación con la educación postsecundaria, esta parte establece como grupo de comparación, para ilustrar mejor la descripción de los "primera generación", aquellos jóvenes que al igual que sus padres lograron acceder a ella.

Finalmente se establecen conclusiones con el objetivo de aportar elementos para abordar los temas de equidad en educación superior desde la política educativa.

\section{Marco conceptual}

Desde un punto de vista conceptual, la idea de "primera generación en educación superior" nos remite a conceptos íntimamente relacionados en el campo educativo como son el de "trayectoria", de "movilidad social" y el de "estrategia". Revisaremos en términos generales algunas aproximaciones de cada uno de ellos.

\section{Trayectoria}

A diferencia de otros conceptos que buscan adentrarse en los recorridos que siguen los sujetos, el de trayectoria "se ubica en el plano social, de las posiciones que van ocupando los sujetos en la estructura, o lo que es igual, en el campo de las relaciones de poder entre los grupos. Para el análisis de trayectorias, no importa la secuencia que forman las sucesivas fases de generación de nuevos individuos adultos, sino las posiciones estructurales y las disposiciones subjetivas que producen -en el doble sentido de 'ser producto de' y 'producir'- esos cambios de condición” (Dávila y Ghiardo, 2005b, p. 118). 
Para hacer más comprensible esta noción, podemos compararla con la de transición. Este sería un proceso inevitable, presente en todo momento histórico y común a todo individuo, ya que "siempre y en todo lugar los niños crecen y se convierten en adultos, más allá de lo que social y culturalmente signifique, de lo que los haga adultos, de los signos y ritos que marquen el paso de una a otra etapa, de la edad que señale la mayoría de edad" (Dávila y Ghiardo, 2005b, p. 118).

En resumen, si para el análisis de las transiciones "el paso de estudiante a trabajador importa en sí mismo, la edad en que se produce es un factor que influye en la descripción de la estructura de las transiciones. Para las trayectorias importan, en cambio, el grupo social de origen, el grado de educación alcanzado, el tipo de establecimiento escolar, el título y el tipo de trabajo al que se accede con él y la valoración social y simbólica de lo obtenido" (Dávila y Ghiardo, 2005b, p. 118).

"Igual que la biografía comienza con el lugar y fecha de nacimiento, las trayectorias sociales tienen un punto de inicio que está definido, en este caso, por el volumen y estructura de capitales con que cuenta un individuo al momento de nacer. En la definición de este punto, la familia es el factor determinante. De ella depende el "patrimonio" de capital heredado, condiciones materiales que determinan la existencia, el lugar donde se vive. Usando como metáfora la idea del "juego", la posición de origen dispone las cartas para jugar, determina el lugar y la "fuerza" con que parte una trayectoria, los caminos posibles de ser recorridos, lo que se puede ser y llegar a ser" (Dávila, Ghiardo y Medrano, 2005, p. 80).

Este tipo de análisis ha sido profundamente trabajado en las teorías sociales denominadas "reproduccionistas". Uno de los autores vinculados con esta perspectiva es Bourdieu quien señala que "a un volumen determinado de "capital heredado" corresponde un "haz de trayectorias" más o menos probables que conducen a unas posiciones más o menos equivalentes - es el campo de los posibles objetivamente ofrecidos a un agente determinado-" (Bourdieu, 1988, p. 108). Nos habla de la existencia de una correlación muy fuerte entre las posiciones sociales y las disposiciones de los agentes que las ocupan. 
Elemento clave para comprender esta perspectiva de trayectoria es el de "habitus", que para Bourdieu es esta suerte "de sentido práctico que ilumina eso que se debe hacer en una situación dada”. (Bourdieu, 1994, p. 45). En este sentido, el habitus se encuentra definido por las condiciones de existencia de los sujetos, y que corresponde a aquellas disposiciones que funcionan como "... principios generadores y organizadores de prácticas y representaciones que pueden ser objetivamente adaptadas a su objeto sin suponer la dirección consciente de sus fines y el dominio expreso de las operaciones necesarias para esperarlas...". (Bourdieu, 1980, pp. 88-89).

Si bien el habitus reproduce las condiciones sociales de las que es producto, también podemos mencionar que "las prácticas disruptivas y transformadoras son posibles cuando el desarrollo de nuevas condiciones históricas generan la oportunidad de reorganizar las disposiciones adquiridas" (Nora Gluz, 2006, p. 60). En este sentido el habitus no sólo comprende un aspecto reproductivo considerando al sujeto como un ser pasivo, sino que reconoce un aspecto reinterpretativo y activo en éste que le permite pensar y actuar el cambio.

\section{Estrategia}

Esta capacidad reinterpretativa y activa de los sujetos sociales puede ser leída también a través de la noción de "estrategia". De esta forma, volviendo a la "metáfora del juego", podemos reconocer que si bien existe un conjunto de coerciones y exigencias en todo "juego social", también existe un "sentido del juego", "que implica la existencia de estrategias que posibilitan la innovación permanente, la posibilidad de adaptarse a situaciones indefinidamente variadas, nunca perfectamente idénticas entre sî" (Baeza, 2009). A juicio de Bourdieu, "el buen jugador, que es en cierto modo el juego hecho hombre, hace en cada instante lo que hay que hacer, lo que demanda y exige el juego" (Bourdieu, 1988, p. 70). En otras palabras, en el pasatiempo de los naipes todos partimos con una combinación de cartas que de alguna forma condiciona (no determina) nuestra posibilidad de éxito en él, pero depende de nosotros lo que podamos hacer con ese capital inicial. Tenemos la capacidad de "leer" nuestro entorno y adaptarnos a él como mejor nos parezca. 
El concepto de "estrategia" tiene su base en el enfoque interaccionista, ya que parte de una concepción del sujeto como un ser activo, consciente y reflexivo, en que su condición de ser humano le permite comprender su situación para reflexionar y actuar en ella. Como dice Woods (1983), la estrategia "interviene en el cruce de la biografía y de la estructura, allí donde la intención personal se encuentra con la coacción (tensión) que impone la sociedad"6 (Woods, 1983. Citado en De Queiroz y Ziolkovski, 1997, p. 19).

Desde esta perspectiva, para Dubet y Martuccelli (1998) el actor construye una racionalidad en función de sus objetivos, recursos y posiciones. La identidad se define no sólo por lo que se es, sino también por lo que se posee y los intereses que se tengan. Así la experiencia aparece como "mercado", como competencia, por lo que la socialización no puede ser entendida sólo como la interiorización de un habitus, sino también como el aprendizaje de una "capacidad estratégica".

Según estos autores, es la propia escuela la que "enseña" a los sujetos a hacer uso de esta capacidad estratégica, sobre todo en un contexto donde el sistema educativo entra en un proceso de masificación y diversificación continua donde las ramificaciones escolares se multiplican generando relaciones jerárquicas entre ellas. No sólo se origina una inflación y devaluación de los diplomas por encontrarse algunos de ellos casi universalmente distribuidos (como sucede hoy con el certificado de enseñanza media), sino que además se jerarquizan tipos de colegios, de instituciones de educación superior y de carreras. Este efecto implica que aquello que el nivel de enseñanza media acreditaba como competencias pierde cada vez más relevancia (se devalúa) en el "mercado de los diplomas", ya que son cada vez más los que acceden a él (Dubet y Martuccelli, 1998).

Es por ello que con la masificación de la educación media, según estos autores, se endurece la competencia educativa y social, obligando a los sujetos a generar estrategias y a utilizar racionalmente

6 Concepto que es utilizado por diversas vertientes, sobre todo con relación al mundo escolar. Por ejemplo, ésta adquiere una dimensión central en la "experiencia escolar" de Dubet y también en los trabajos de Boudon y su "actor racional" para comprender las desigualdades escolares. 
el sistema educativo. Con ello la idea de proseguir estudios superiores se presenta muchas veces como una obligación si se pretende posicionarse de manera no precaria en el mercado laboral.

Es importante resaltar la noción de estrategia ya que es ella la que posibilita imaginar una figura escolar distinta de la de "los herederos" y "los reproductores" en la nomenclatura de Bourdieu. En el sistema escolar es posible observar trayectorias de sujetos que escapan a la norma, ya que no estamos determinados por nuestro medio. Contamos con herramientas para resistir la reproducción social.

\section{Movilidad social}

A primera vista, sería posible decir que aquellos jóvenes "primera generación en la educación superior" adquieren notoriedad social ya que han logrado romper la ya muy sabida "reproducción social" (intergeneracional) que, según algunos autores, es posible observar en el sistema educativo. Lo que es importante de mencionar considerando que hoy la movilidad social se encuentra muy supeditada al tipo de educación que se recibe y al desempeño que se logre tener en este espacio.

Pero si se hace un análisis más profundo de esta situación nos encontramos con que la masificación de la educación media pierde valor en el mercado de la empleabilidad. En este sentido acceder a la educación superior puede ser visto como un proceso obvio, casi natural en el contexto del Chile actual, sobre todo en la lógica de poder establecer en dicho sistema una "distinción", que en este momento pasa por acceder a este tipo de enseñanza que es capaz de marcar la diferencia en el mundo laboral.

Pero, ¿cuánta "diferencia" o "distinción" genera acceder a la educación superior? Los datos que entrega la última encuesta CASEN dan pistas de ello. Según ésta existe una relación positiva, pero no proporcional, entre los años de escolaridad y el ingreso promedio mensual de un trabajador. Existe un aumento moderado del ingreso promedio mensual por ocupación principal entre los cero y los quince años de escolaridad, en la mayoría de los casos, siendo el punto de inflexión los dieciséis años de escolaridad (es decir, en el $4^{\circ}$ año de 
educación superior), desde donde se puede apreciar un aumento considerable del nivel de ingreso (CASEN, 2006). En el fondo, la "distinción" más marcada se empieza a configurar con carreras que llevan a un título profesional y no técnico necesariamente.

Figura 1. Salario promedio por años de escolaridad. Pesos 2006

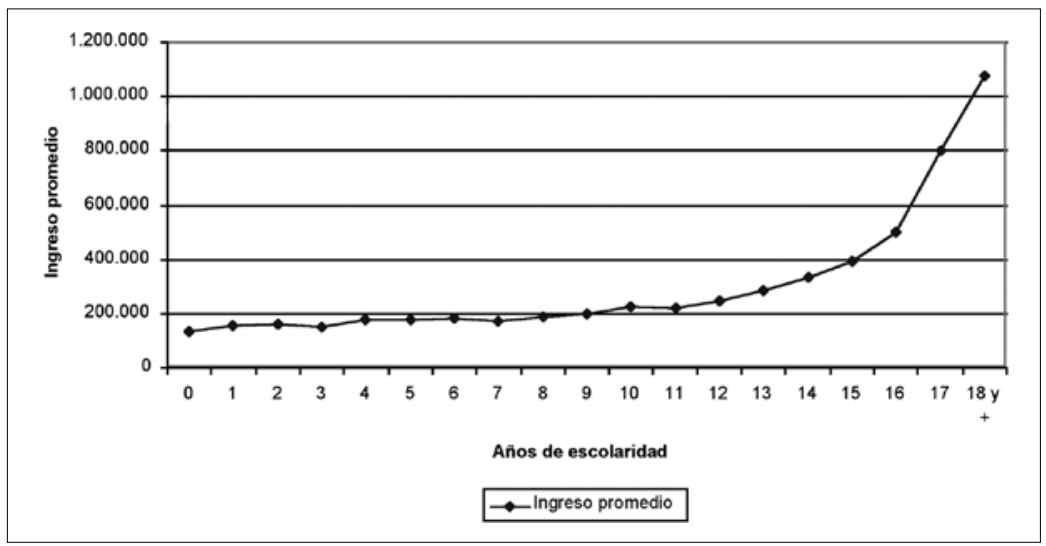

Fuente: CASEN, 2006.

Trayectorias, sistema educativo y primera generación en educación superior

Es posible reconocer en este marco conceptual el nuevo fenómeno de la "primera generación en la educación superior", ya que en definitiva y considerando la idea de "estrategia", el concepto de "trayectorias" puede ir más allá que la simple relación entre condición social de origen y su "determinista" proyección en cuanto al punto de llegada de los sujetos.

Por ejemplo, algunos autores, reflexionando sobre las trayectorias educativas de los estudiantes de secundaria muestran la importancia de las "expectativas y planes que los estudiantes y sus padres -en conjunto con las escuelas- van poniendo en juego para definir sus prácticas, sus acciones" (Montes y Sendón, 2006, p. 390), estableciéndose un vínculo claro "entre la situación (posibilidades de inserción laboral y/o de seguir diferentes estudios terciarios, tipos de capital con que se cuenta familiarmente para ello, entre otros.) y la disposición (gusto por el estudio, tradición familiar por cierto 
estudio, cultura del trabajo en que se socializaron los estudiantes). En este vínculo ninguno de los términos es determinante sino que (...) en el juego de estos elementos es donde se podrán caracterizar la o las prácticas a desarrollar por los estudiantes secundarios" (Montes y Sendón, 2006, p. 390).

Es evidente que la educación es el lugar privilegiado -por sus objetivos institucionales y definición de tiempos y espacios- para diseñar la construcción de proyectos de vida de los estudiantes (Guichard, 1993). Estos contemplan definiciones de trayectorias educativo-laborales considerando las necesidades y expectativas de los propios jóvenes.

Así, las trayectorias y proyectos de vida están atravesados por diversos factores. Aquello que define lo que los estudiantes quieren hacer y realizar una vez fuera del liceo se mueve entre la intencionalidad y los méritos personales y aquellas condiciones estructurales que determinan las opciones que los sujetos tienen al momento del egreso, y también durante su estadía escolar. Así entendido, no todos los estudiantes viven su escolaridad de la misma manera y tampoco el momento del egreso; lamentablemente sigue siendo una característica más o menos extendida entre los jóvenes de sectores populares el que experimenten el final de su trayectoria escolar como un momento liminal en que aquello que el liceo presentaba como aspiraciones (una mejor educación, trabajo, mejores condiciones para enfrentarse a la vida) se vuelven muchas veces promesas incumplidas (Dávila et al., 2006; Goicovic, 2002; Weinstein, 2001). Lo interesante es que progresivamente los anhelos por continuar estudios superiores se están haciendo realidad, incluso en los segmentos más pobres de la población, lo que como fenómeno creemos requiere ser estudiado en profundidad para aportar insumos a las políticas educativas que lo sustentan.

\section{Metodología}

Este estudio se basa en la Encuesta Preliminar de Estudiantes 2009 realizada por el Programa de las Naciones Unidas para el Desarrollo, PNUD, en conjunto con el Ministerio de Educación con 
representatividad nacional y con el objeto de estudiar las trayectorias de una muestra de jóvenes desde segundo medio el 2003 hasta el año 2009, cuando la mayoría tenían cuatro años de egreso. Por lo tanto, mediante esta encuesta es posible conocer qué han hecho los jóvenes después de la enseñanza media, ya sea que hubieran o no continuado sus estudios.

Fueron entrevistados 1.599 jóvenes a través de todo el país, a los cuales se les aplicaron ocho módulos para conocer sobre sus historias educacionales (educación básica, media y superior), capacitaciones, trayectoria laboral, salud, situación personal, información de sus hogares, características de la vivienda y habilidades generales; a esta información fueron agregados datos del Registro de Estudiantes de Chile (REC) desde segundo medio hasta el momento de abandonar la enseñanza escolar (ya sea que egresaran o no de cuarto medio). Además, los puntajes y cuestionarios de la prueba SIMCE de segundo medio 2003 y los resultados PSU de quienes la rindieron el 2005 y/o 2006.

Dada esta unión de bases de datos, la temporalidad de todas las variables no es la misma, considerando además que en la Encuesta Preliminar de Estudiantes hay preguntas que buscan reconstruir las historias educacionales y conocer los motivos de ciertas decisiones tomadas en momentos anteriores.

Como indicador de la educación de los padres se empleó el máximo nivel educativo del padre y la madre reportado en el Cuestionario de Padres y Apoderados de la prueba SIMCE realizada por la muestra en segundo medio 2003. Se asume que la escolaridad reportada de ellos es representativa incluso del nivel educacional de generaciones anteriores, por lo tanto, los jóvenes identificados como "primera generación" son los primeros en acceder a educación superior.

De los 1.599 jóvenes encuestados, sólo se dispone de información de la educación de los padres en 1.378 casos y si son excluidos los estudiantes que en su trayectoria educativa abandonaron la educación media, entonces se obtiene un universo de 1.315 entrevistados. De ellos un $75 \%$ tiene padres con educación media completa o menos (1.042), el resto cuenta con estudios de educación superior. 
El estudio se realiza sobre la base de la comparación de tres grupos principales ${ }^{7}$, los que se estructuran en relación con la educación máxima de los padres y encuestados. Estos son:

- "Jóvenes primera generación": que acceden a la educación superior teniendo padres sin haber cursado este tipo de educación. Este grupo equivale a $41 \%$ del total de los entrevistados.

- "Jóvenes reproductores": que si bien egresaron de la enseñanza media no lograron acceder a la educación superior con padres sin educación terciaria. Este grupo equivale al 34,1\% del total.

- "Jóvenes herederos": que al igual que sus padres lograron acceder a la educación superior. Este grupo equivale al 22,6\% del total.

Existe un grupo de entrevistados que no han accedido a la educación superior y tienen padres con más de doce años de escolaridad, sin embargo, equivalen sólo al 2,3\% y no constituyen un grupo significativo para el análisis posterior.

El objetivo de diversificar en "grupos de jóvenes" se debe a los diferentes tipos de análisis que se requieren realizar con el fin de caracterizar bien a aquellos que pertenecen a la primera generación en educación superior. Las primeras secciones del apartado IV tienen por finalidad caracterizar a los jóvenes primera generación e identificar las variables que los distinguen respecto a su relación y experiencia con el mundo escolar, para lo cual la comparación relevante se estructura respecto al grupo de jóvenes que, como sus padres, comparten la característica de no poseer educación superior. En la sección siguiente, el objetivo es identificar las motivaciones y acciones realizadas para acceder a la educación superior, por ello se realiza la comparación sobre los tres grupos para conocer qué diferencia a éstos cuando todos lograron egresar de la enseñanza media en una misma cohorte. Finalmente, en las últimas secciones, el objetivo es comparar a los jóvenes primera generación con aquellos definidos como "herederos" con el objeto de conocer si hay diferencias entre ellos o elementos de ventaja de un grupo sobre otro a pesar de tener niveles de escolaridad similares.

7 El nombre que ha sido utilizado para cada grupo toma como referente los conceptos claves utilizados por Pierre Bourdieu en su análisis sobre la reproducción generada por el sistema educativo. 


\section{Caracterización de los jóvenes que pasan a ser primera generación de estudiantes en la educación superior}

El grupo de jóvenes primera generación en educación superior equivale a un $41 \%$ del total de los entrevistados, lo que del total de los que ingresan a la educación superior corresponde al 64,4\%.

En este tipo de estudios, una de las aristas importantes son las relaciones de género, aunque no es uno de los objetivos de este estudio. Sin embargo, de este grupo se puede mencionar que no se observa que exista una importante diferencia a este respecto, ya que en el caso de los hombres que ingresan a la educación superior un $55,8 \%$ de ellos es primera generación, mientras que en el caso de las mujeres esta proporción llega al 53,5\% del total de ellas.

Ha existido desde hace tiempo un importante esfuerzo para establecer relaciones igualitarias en el sistema escolar. Dado esto, es probable que los resultados observados sean fruto de este esfuerzo, en donde similares proporciones de hombres y mujeres tienen la oportunidad de dar un salto significativo educacional respecto de sus familias, en un contexto donde las mujeres han venido ganando terreno fuertemente en los últimos años.

\section{IV.1. "La combinación inicial de cartas y el apresto para el} juego": caracterización sociocultural de los jóvenes primera generación

Partimos describiendo las condiciones socioculturales de origen de este tipo de estudiantes respecto a la composición familiar de los hogares de ellos cuando se encontraban en segundo medio el año 2003. Es importante destacar que en este caso nuestro universo son los jóvenes que cuentan con padres con máximo doce años de escolaridad.

Las variables referidas a la composición familiar son de interés, ya que son mencionadas en variados estudios respecto de los efectos en los niveles de escolaridad logrados por hijos de familias de diferente composición, tal como la presencia de hermanos mayores o menores o de uno o ambos padres (Sanhueza, 2009, y SITEAL, 2006). 
Por ejemplo, si se observa a los jóvenes que cuentan por lo menos con un hermano, una mayor proporción de éstos se concentran en el grupo de la "primera generación" (57,3\%), en comparación con los "jóvenes reproductores" donde sólo este tipo de composición familiar llega al 42,7\%. Si bien la información recolectada no permite discriminar si estos son hermanos mayores o menores, se pueden establecer ciertas hipótesis que permiten explicar esta situación a la luz de diferentes estudios. Estos teorizan sobre los efectos de los hermanos sobre los logros educacionales, pudiendo haber efectos positivos o negativos según el sexo de ellos o si son mayores o menores ${ }^{8}$.

Otro elemento de caracterización de la familia es si el hogar del alumno estaba compuesto por ambos padres cuando se encontraba en segundo medio. Un $68,2 \%$ de los hogares posee esta particularidad; no obstante, es posible observar diferencias importantes en cuanto al acceso a educación superior. Hay una mayor proporción de estudiantes primera generación en hogares que reportan la presencia de ambos padres, 58\%, en comparación del 47,5\% donde sólo se encuentra uno de ellos.

Respecto a esto, las hipótesis posibles de plantear para explicar que esta condición ofrezca más posibilidades a los hijos para continuar con la educación superior puede ser que:

a) en un hogar con dos padres es posible que ambos trabajen, aumentando los ingresos del hogar;

b) es menor la posibilidad de sustitución de tareas, donde un hijo o hija debe comprometerse con alguno de los papeles del adulto ausente (como el cuidado del hogar o el trabajo remunerado).

8 Los estudios que muestran efectos positivos se fundamentan en lógicas distintas: Hay estudios, por ejemplo, que muestran que cuando el hermano es mayor y logra ingresar a la educación superior éste sirve de modelo a seguir por parte de los hermanos menores. Hay otros análisis que han mostrado cómo hay familias que "sacrifican" las trayectorias educativas de algunos de sus miembros en pos de darles la oportunidad a otros. Este sacrificio se encarna en tener que optar por el mundo del trabajo o de los quehaceres del hogar. Los primeros análisis han sido utilizados para cuestionar la generalización que evidencian los análisis "simplistas" que relacionan escolaridad de los padres con logros educativos de los hijos (Charlot, 1997), a diferencia de los segundos que surgen de estudios que han indagado en la deserción escolar (Instituto Nacional de la Juventud, 2002). 
Otro elemento propio de la familia, y que ha sido vastamente estudiado en términos de evidenciar el capital cultural de los estudiantes, son los años de escolaridad promedio de los padres. Los datos indican que existe una diferencia significativa entre los años de escolaridad promedio de los padres de los jóvenes primera generación y quienes no logran serlo, considerando que ambos grupos tienen padres con a lo más doce años de escolaridad. Los padres de los jóvenes primera generación poseen en promedio 10,7 años de escolaridad a diferencia de los no lo logran que tienen 9,2 años.

Una manera distinta de hacer este mismo estudio es analizando el grado de escolaridad de los padres. Con ello se observa que efectivamente a mayor escolaridad de los padres, mayor es la proporción de estudiantes que logra acceder a la educación superior. Así, entre los jóvenes que tienen padres con educación básica incompleta la proporción de éstos que logran ingresar a la educación superior es de sólo un 32\%, entre quienes tienen la educación básica completa es de $41,6 \%$, para los padres con enseñanza media incompleta esta llega al $49 \%$ y finalmente, entre los padres que lograron completarla la proporción llega al 69\%.

Tabla n. ${ }^{\circ}$ 1. Porcentaje alumnos primera generación según máxima educación de sus padres.

\begin{tabular}{|l|c|c|c|}
\hline Máxima educación padres & Primera generación & Reproductores & Total \\
\hline Educación básica incompleta & 31,9 & 68,1 & 100 \\
\hline Educación básica completa & 41,7 & 58,3 & 100 \\
\hline Educación media incompleta & 49,1 & 50,9 & 100 \\
\hline Educación media completa & 69 & 31 & 100 \\
\hline
\end{tabular}

Fuente: elaboración propia. EPE 2009.

Otra variable que es muy utilizada como indicador de capital cultural de las familias son el número de libros en el hogar. Los datos indican que el año 2003, cuando los estudiantes cursaban segundo medio, en los hogares de los jóvenes que ahora son primera generación había una mayor cantidad de textos que en los hogares de quienes no accedieron a la educación superior. Si se observa la distribución de textos por tramos para cada grupo, en el $43 \%$ de los hogares de los jóvenes primera generación había entre once y cincuenta libros y un 22,6\% tenía más de cincuenta y uno, mientras que en los hogares 
del otro grupo, en el 44\% de ellos había sólo de uno a diez libros y aquellos hogares que tenían cincuenta y uno o más libros sólo llegaban al $12 \%$.

Una variable más subjetiva, pero que está muy validada en los análisis educativos, son las expectativas de los padres respecto al logro educativo de sus hijos, llegando a identificarlas como un elemento determinante tanto en los resultados de aprendizaje (los análisis al SIMCE así lo demuestran) como en el acceso a la educación superior (Concha, 2009). Respecto a los datos, la tabla n. ${ }^{\circ} 2$ muestra cómo las expectativas de los padres, respecto al nivel de educación que alcanzarán sus hijos (declaradas en el cuestionario SIMCE 2003), se distribuyen de manera diferente según el nivel de educación que finalmente han alcanzado sus hijos (si lograron acceder o no a la educación superior). Mientras mayor sea el nivel educacional que esperan alcancen sus hijos, mayor es la proporción de ellos que es primera generación.

Tabla $n{ }^{\circ}$ 2. Porcentaje de jóvenes primera generación por expectativas de los padres

\begin{tabular}{|l|c|c|c|}
\hline Expectativas sobre educación alcanzada por hijos & Primera generación & Reproductores & Total \\
\hline Educación media & 22,4 & 77,6 & 100 \\
\hline Educación CFT o IP & 50,8 & 49,2 & 100 \\
\hline Educación universidad & 80,3 & 19,7 & 100 \\
\hline
\end{tabular}

Fuente: SIMCE y EPE 2009.

Una caracterización del ingreso de los hogares de los estudiantes durante su segundo medio el año 2003, consultados en el cuestionario SIMCE, indica que no había una diferencia significativa entre los hogares de los jóvenes primera generación y quienes no lo son. La mayoría se concentra en el tramo de 200.000 a 300.000 pesos mensuales, en un $41 \%$ para el caso de los primeros y $47 \%$ para los hogares de los jóvenes reproductores. Por su parte, en los tramos más bajos y superiores las diferencias son mínimas.

Por otra parte, las oportunidades educacionales no están distribuidas homogéneamente entre las comunas del país. En las pequeñas no están disponibles todos los niveles educacionales, así, muchos jóvenes deben emigrar a otras comunas para proseguir sus estudios. Considerando ello, es posible realizar un análisis por tamaño 
de las comunas, a través de las residencias declaradas en segundo medio por los jóvenes.

Las comunas declaradas fueron clasificadas en: Región Metropolitana, Grandes Ciudades (con más de 150.000 habitantes) y Ciudades Pequeñas (con población menor a 150.000 habitantes). Los datos arrojan dos resultados de interés. Primero, que hay una mayor concentración de padres con doce años de escolaridad o menos en las ciudades con menos de 150.000 habitantes. Es decir, que cerca del $87 \%$ de los padres que vivían en Ciudades Pequeñas a lo más había completado la enseñanza media, en comparación con la Región Metropolitana y las Grandes Ciudades con un 68\% en cada una de ellas. Segundo, a pesar del resultado anterior, las Ciudades Pequeñas muestran una significativa reducción en la diferencia educacional por tamaño de las comunas (mirado intergeneracionalmente). Así, el porcentaje de quienes son primera generación en cada una de ellas respecto del total que vivían en ellas, muestra que en éstas la tasa es de 47,6\% para las Ciudades Pequeñas, mientras que en las Grandes Ciudades es de 62,5\% y en la Región Metropolitana es de un 58,5\%. Estos resultados parecen indicar que a pesar de que inicialmente las oportunidades estaban geográficamente muy desequilibradas, en cuanto a la educación promedio de los padres de los jóvenes, se observa que ahora la proporción de quienes logran dar el salto a la educación superior ha aumentado en mayor medida en las Ciudades Pequeñas (en este caso analizado a sus hijos). Antes el lugar de residencia influía más en la educación que se podía alcanzar, al parecer eso es cada vez menos determinante.

IV.2. "Primeras jugadas en el espacio escolar": desempeño y trayectorias escolares de los jóvenes primera generación en educación superior

Las trayectorias educacionales de los jóvenes primera generación son presentadas a continuación y en comparación con aquellos que teniendo a padres de iguales características no lograron ingresar a la educación superior.

Es posible establecer que la mayoría de los jóvenes primera generación en su infancia asistieron a prekinder $(62,5 \%)$, mientras 
que el porcentaje que asistió a este tipo de educación de aquellos que no lograron serlo sólo fue un 49,7\%. Este dato corrobora hallazgos de otros estudios que muestran las ventajas que tiene ingresar tempranamente a la educación formal en términos de los logros educativos posteriores (Contreras et al., 2007).

En términos del tipo de institución educativa que cursan, la mayoría de los estudiantes primera generación proviene de establecimientos municipales $(47,4 \%)$, en segundo lugar de particulares subvencionados $(40,3 \%)$, luego las corporaciones privadas $(8 \%)$ y una porción muy pequeña desde los particulares pagados $(4,3 \%)$. Estos resultados pueden estar influenciados por la distribución no aleatoria de los padres con educación media o menos. El 54\% de ellos matriculó a sus hijos en establecimientos municipales, el 33,6\% en particulares subvencionados, un 10\% en corporaciones privadas y sólo un $2 \%$ en particulares pagados. Por este motivo, puede ser conveniente prestar atención a un indicador de eficiencia de cada dependencia. En este sentido es posible observar el porcentaje de estudiantes que accede a la educación superior respecto del total de alumnos en cada dependencia. Los resultados se presentan en la tabla n. ${ }^{\circ} 3$, donde se observa que la eficiencia es mayor en los establecimientos particulares subvencionados ${ }^{9}$.

Tabla n. ${ }^{\circ}$ 3. Porcentaje de alumnos primera generación por dependencia.

\begin{tabular}{|l|c|c|c|}
\hline Dependencia & Primera generación & Reproductores & Total \\
\hline Municipal & 47,7 & 52,3 & 100 \\
\hline Particular subvencionado & 65,4 & 34,6 & 100 \\
\hline Particular pagado & 100 & 0 & 100 \\
\hline Corporación privada & 44,3 & 55,7 & 100 \\
\hline
\end{tabular}

Fuente: elaboración propia. EPE 2009.

Respecto del tipo de enseñanza del establecimiento donde cursaban segundo medio los jóvenes primera generación, el 70,6\% estudió en establecimientos educativos modalidad H/C; sin embargo,

9 Es importante destacar que en el caso de los establecimientos particulares pagados, se observa un resultado del $100 \%$, sin embargo este valor no puede ser tomado como representativo, dada la escasa representación de los padres con educación media o menos en estos establecimientos. 
esto está probablemente más relacionado con que la mayoría de los estudiantes asiste a establecimientos que imparten esta modalidad. Siguiendo el análisis de eficiencia, se observa que de todos modos esta es mayor en la modalidad H/C $(62,6 \%)$ que en la T/P $(41,6 \%)$, a pesar del incremento progresivo de expectativas y continuación de estudios de los jóvenes que cursan esta última modalidad de enseñanza ${ }^{10}$.

En cuanto al rendimiento académico, es posible identificar tres aproximaciones del desempeño de los jóvenes durante su trayectoria en la enseñanza básica y media. Estas son: repitencia, ranking de promedios generales y puntaje en la prueba SIMCE segundo medio 2003.

En cuanto a la repitencia, es posible establecer un cruce de información de tal modo de conocer si los jóvenes encuestados repitieron algún curso entre la enseñanza básica y media, aunque no el número de veces de ocurrencia. De este modo, los datos indican que los jóvenes primera generación muestran que cerca del 85\% de ellos no repitió durante toda la enseñanza obligatoria. Por el contrario, los jóvenes reproductores muestran una proporción menor (66\%).

Respecto al desempeño en la prueba SIMCE de segundo medio (2003), es posible observar que los jóvenes primera generación tienen un puntaje estadísticamente mayor que quienes no accedieron (252 puntos promedio versus 225). Lamentablemente esta superioridad no significa necesariamente el haber desarrollado aprendizajes de calidad, ya que los jóvenes primera generación sólo logran obtener en promedio la media nacional del SIMCE ${ }^{11}$.

La misma tendencia es observada en el ranking de egreso (percentiles) respecto de sus promedios generales obtenidos en el último curso realizado al egresar de la enseñanza media, donde los jóvenes

10 Ver Informes de Centro Microdatos (2009) y MINEDUC (1998). De estos informes se desprende que ha existido un aumento de la proporción de estudiantes T/P que ingresa a la educación superior. Según el segundo estudio, para los egresados de 1997, un 30\% de ellos accedió, mientras que para los egresados del 2008 esta proporción llegó al 47\%, según el informe más reciente.

11 Por ejemplo, los jóvenes herederos logran como promedio SIMCE los 295 puntos, superando en más de 40 puntos a los de primera generación. 
primera generación están en promedio en el percentil 55, mientras que su grupo complementario (estudiantes reproductores) en el 41.

Un análisis de los gastos relacionados con educación, colegiatura y otros no relacionados con este ítem, consultados en el cuestionario SIMCE segundo medio 2003, muestra que no existían diferencias significativas entre los hogares de los jóvenes primera generación y los reproductores. En general, la mayoría no pagaba por la colegiatura de sus hijos, 37\% y 42,6\% para cada grupo. Y en el caso de los gastos en educación, la mayoría de ambos grupos gasta mensualmente en promedio entre cinco y diez mil pesos, en un $32 \%$ y $36 \%$ respectivamente. Esto señala que las diferencias entre ambos grupos no radican en los niveles de inversión de ambos.

En resumen, podemos decir que los jóvenes que logran ser primera generación en la educación superior presentan una experiencia escolar más "adaptada" a los códigos y valores que la cultura escolar manifiesta como claves para el éxito escolar. Al parecer estrategias como ejercer un mayor aprovechamiento escolar, traducido en una mayor promoción de niveles educativos, mejores notas y mejor puntaje SIMCE, nos hablan de ello.

IV.3. Estrategias desplegadas para ganar la primera partida: predisposiciones, estrategias y racionalidades puestas en juego en el ingreso a la educación superior por parte de los jóvenes primera generación

Al salir de la enseñanza media, los jóvenes se sienten obligados a tomar una decisión, socialmente definida como "trascendental" en la vida de las personas, ya que enmarcaría fuertemente el trayecto que se seguirá en el futuro. El acto de decidir se torna central en este proceso de tránsito.

A este respecto, cuando se les preguntó a los entrevistados respecto a lo que querían hacer al momento de egresar de la enseñanza media, un 78\% de los jóvenes primera generación, un 64\% de los jóvenes reproductores y $80 \%$ de los jóvenes herederos señalaron que tenían claro qué hacer. Respecto de la actividad que querían 
realizar, la mayoría deseaba seguir estudiando, ver tabla n. ${ }^{\circ} 4$, ya sea con dedicación exclusiva o trabajando al mismo tiempo. En un alto porcentaje, aquellos que declararon que querían seguir estudios posteriores tenían claro la carrera a seguir, $82 \%$ de los que son primera generación, $83 \%$ de los reproductores y un 79\% de los herederos. En cuanto a la institución donde realizar estos estudios, tanto los primera generación como los herederos declararon haber preferido en ese entonces una universidad tradicional, $40 \%$ los primeros y $60 \%$ los segundos. En cambio en los jóvenes reproductores esta proporción baja al 26\%, compartiendo preferencias con los institutos profesionales.

Tabla n. ${ }^{\circ}$ 4. Distribución de respuestas: ¿qué quería hacer al egresar de 4to medio?

\begin{tabular}{|l|c|c|c|c|}
\hline ¿Qué quería hacer? & Primera generación & Reproductores & Herederos & Total \\
\hline Estudiar & 80,19 & 39,36 & 94,96 & 71.67 \\
\hline Estudiar y trabajar & 15,75 & 21,28 & 2,94 & 14,16 \\
\hline Trabajar & 3,1 & 35,11 & 0.84 & 12,14 \\
\hline Otro & 0,96 & 4,25 & 1,26 & 2,03 \\
\hline Total & 100 & 100 & 100 & 100 \\
\hline
\end{tabular}

Pero para acceder a la educación superior no sólo se requieren las ganas, sino también, en la mayoría de los casos, rendir la PSU con un buen desempeño, que facilite la probabilidad de ingresar y con recursos que permitan costear dichos estudios. En el fondo, además de las intenciones se requiere contar con ciertas condiciones que lo hagan posible, que para algunos puede significar "tenerlo prácticamente asegurado", mientras que para otros significa el diseño e implementación de estrategias que lo proporcionen.

El hecho de dar o no la PSU, como el modo definido de prepararse para rendirla, nos habla de estrategias que son utilizadas por los jóvenes para mejorar sus conocimientos. Respecto a lo primero, el 80,7\% de los "jóvenes primera generación" rindió la PSU, porcentaje que contrasta con el 32,8\% de los "jóvenes reproductores", aunque cercano al 97,3\% de los "jóvenes herederos". Con relación a lo segundo, los de primera generación se prepararon principalmente en un preuniversitario (39\%) y solo o en su establecimiento educacional (25\% y $24 \%$ respectivamente); similar distribución a los jóvenes herederos (vía preuniversitario 53\%, solo 24\% y en del colegio 16,5\%). En cambio, el grupo de los jóvenes reproductores se 
preparó en su colegio (32\%), un 24\% lo hizo solo y un $18 \%$ lo hizo en preuniversitarios. En una elevada proporción el preuniversitario era pagado en los tres grupos: siendo los jóvenes primera generación y los herederos los que más "invirtieron" en ello -84,6\% y 95,5\% respectivamente-, a diferencia de los jóvenes reproductores donde sólo un 50\% lo hizo.

Al comparar las medias de los puntajes promedios (matemática y lenguaje) obtenidos en la PSU rendida entre los años 2005 o 2006 se observan diferencias significativas en éstos de los grupos analizados (tabla n. ${ }^{\circ}$ 5). Los jóvenes primera generación obtienen resultados superiores que el grupo de los jóvenes reproductores, aunque significativamente inferiores que el grupo de los herederos, ya que apenas la mitad de los primera generación obtiene el puntaje mínimo para postular a las universidades del Consejo de Rectores.

Tabla n. ${ }^{\circ}$. Porcentaje estudiantes y puntaje promedio Prueba de Selección Universitaria.

\begin{tabular}{|l|c|c|c|c|c|}
\hline & Ptje. Promedio & $>=450$ & $>=500$ & $>=600$ & $>=700$ \\
\hline & & $(\%)$ & $(\%)$ & $(\%)$ & $(\%)$ \\
\hline Primera generación & 459,5 & 51,6 & 32,9 & 6,7 & 0,7 \\
\hline Jóvenes reproductores & 406,8 & 31,4 & 11,9 & 0,8 & 0 \\
\hline Jóvenes herederos & 558,7 & 82,5 & 70,9 & 37,5 & 8,4 \\
\hline
\end{tabular}

Finalmente, si se observa el tipo de recursos con los que se cuenta para costear los estudios superiores, respecto de los jóvenes primera generación, se observa que financian sus estudios principalmente con el apoyo de su familia (49\%) y el crédito universitario (23\%), relativamente similar al de los herederos $(68 \%$ y $16 \%$ respectivamente). No obstante, se evidencia una diferencia respecto a este último grupo, ya que en los jóvenes primera generación los aportes propios tienen una mayor importancia ( $16,4 \%$ versus un $4 \%$ de los herederos) ${ }^{12}$.

12 Sobre este punto es importante mencionar que en Chile "los costos de los estudios es considerablemente más alto que en los países OCDE que cobran aranceles", como lo señala el informe de la OCDE sobre "La Educación Superior en Chile" del año 2009. Este informe nos muestra, por ejemplo, que si se compara el nivel de los aranceles con el ingreso per cápita del país, Chile es casi dos veces más caro que Corea y tres veces más que Japón, Estados Unidos y Australia. 
Esta información se condice con aquella que muestra la situación laboral, al momento de la encuesta, en que se encuentran los jóvenes investigados. Los jóvenes primera generación son aquellos que mencionan en mayor proporción estar o haber estado trabajando, y al comparar a éstos respecto a los otros grupos, se observa que son ellos quienes presentan la mayor tasa de quienes trabajan y estudian a la vez, un $22 \%$ en el caso de los primera generación y $18 \%$ en el caso de los herederos. De igual modo, en cuanto a la búsqueda de empleo, los primeros llegan al 5\% y 1\% para los segundos.

Claramente, existen elementos que se pueden identificar como estrategias, seguidas por los jóvenes primera generación, que se diferencian respecto de sus pares. Tales como la rendición y preparación de la Prueba de Selección Universitaria, PSU (invirtiendo más recursos en preuniversitarios, por ejemplo), el tener los objetivos claros al momento de egresar y la provisión propia de recursos para mejorar su capital educativo, empleándose para ello. Sin embargo, el proceso estratégico comienza incluso antes de segundo medio, por ejemplo cambiándose de establecimiento durante el paso de la enseñanza básica a la media, aunque dada la estructura de los datos no es posible seguir las trayectorias anteriores en el pasado de los jóvenes.

\section{IV.4. Respecto al lugar del podio logrado luego de la} primera partida: tipo de posicionamiento en la educación superior de los jóvenes primera generación

Cerca de la mitad de los jóvenes primera generación ingresó a estudiar a una universidad, ya sea privada o tradicional, aunque un porcentaje no menor optó por un instituto profesional. A diferencia de los jóvenes herederos, donde un $81 \%$ optó por universidades (tabla n. ${ }^{\circ}$ 6).

Tabla n. ${ }^{\circ}$ 6. Instituciones de acceso a educación superior

\begin{tabular}{|l|c|c|}
\hline Institución educación superior & Primera generación & Jóvenes herederos \\
\hline Universidad privada & 28,4 & 42,3 \\
\hline Universidad tradicional & 19,3 & 38,6 \\
\hline CFT & 16,4 & 4,7 \\
\hline IP & 35,9 & 14,4 \\
\hline Total & 100 & 100 \\
\hline
\end{tabular}


Además, es posible establecer diferencias en las instituciones a las que acceden los jóvenes primera generación según el nivel de escolaridad de sus padres. A mayor escolaridad de éstos mayor es la proporción de jóvenes que ingresa a instituciones que requieren más años de estudios para adquirir una certificación ${ }^{13}$ (tabla n. ${ }^{\circ}$ 7).

Tabla n. ${ }^{\circ}$. Porcentaje estudiantes primera generación matriculados en instituciones de educación superior según máxima escolaridad de los padres

\begin{tabular}{|l|l|c|c|c|}
\hline Máxima ed. padres & CFT & IP & Universidad & Total \\
\hline Educación básica incompleta & 28,9 & 37,8 & 33,3 & 100 \\
\hline Educación básica completa & 11,4 & 51,4 & 37,1 & 100 \\
\hline Educación media incompleta & 18 & 35,1 & 46,9 & 100 \\
\hline Educación media completa & 15 & 32,4 & 52,6 & 100 \\
\hline
\end{tabular}

Fuente: elaboración propia. EPE 2009.

Profundizando más aún, de la tabla n. ${ }^{\circ} 8^{14}$ se desprende que los jóvenes que son primera generación poseen preferencias más concentradas en ciertas áreas disciplinarias en relación al resto de estudiantes que accedieron a la educación superior. Los primeros prefieren en mayor medida carreras del área de Administración y Comercio, Educación y Tecnología. Asimismo, son notorias las diferencias entre éstos y los jóvenes herederos en las preferencias por las áreas de Salud y Ciencias Sociales.

13 Las carreras impartidas en las universidades y en los institutos profesionales que entregan títulos profesionales tienen duraciones mayores ( 4 o 5 años) que aquellas certificaciones técnicas que entregan los centros de formación técnica ( 3 años).

14 Según las carreras declaradas en la encuesta y clasificadas según la información disponible en el sitio web del Consejo Nacional de Educación. www.indices.cned.cl 
Tabla n. ${ }^{\circ}$ 8. Porcentaje de preferencia de áreas de estudios de estudiantes entrevistados.

\begin{tabular}{|l|c|c|}
\hline Áreas carreras & Primera generación & Jóvenes herederos \\
\hline Administración y Comercio & 18,1 & 13,4 \\
\hline Arte y Arquitectura & 4,6 & 7,7 \\
\hline Ciencias & 0,4 & 3,0 \\
\hline Ciencias Sociales & 4,8 & 13,1 \\
\hline Derecho & 6,9 & 5,7 \\
\hline Educación & 28,6 & 17,1 \\
\hline Humanidades & 1,0 & 0,3 \\
\hline Recursos Naturales & 3,1 & 4,0 \\
\hline Salud & 9,0 & 14,8 \\
\hline Tecnología & 23,8 & 20,8 \\
\hline Total & 100 & 100 \\
\hline
\end{tabular}

Fuente: elaboración propia. EPE 2009.

Un elemento que resalta a simple vista es el hecho de que cerca de un estudiante de cada cuatro que son primera generación en educación superior cursa estudios de pedagogía. Educación es el área de mayor adhesión por parte de estos nuevos alumnos ${ }^{15}$.

Finalmente, es posible establecer una relación entre las carreras declaradas por los encuestados y los datos provenientes del sistema de información "Futuro Laboral"16, para obtener una proyección de los salarios promedio futuros que percibirán los jóvenes que se encuentran estudiando o egresaron de una carrera de educación superior al quinto año de egreso aproximadamente. En promedio, sin distinguir por tipo de institución (universidad, IP o CFT), se observa que los jóvenes primera generación obtendrían un salario significativamente menor que los "herederos", es decir, 717.000 pesos mensuales en comparación con el de 1.106.000 pesos mensuales de estos últimos. Esta diferencia se mantiene si se considera sólo a aquellos que estudian en una universidad. Los jóvenes primera generación que estudian en estas instituciones recibirían un salario promedio de 868.000

15 Si bien los estudiantes primera generación ingresan a sus carreras de preferencia con menores puntajes que aquellos que no lo son, la preocupación se establece al ver que los estudiantes que entran a estudiar pedagogía lo hacen con un puntaje promedio de 442 puntos en la PSU, puntaje que evidencia un bajo nivel de logro educativo. Según algunos expertos se torna imposible de subsanar en los 4 o 5 años de estudio que se exige, reproduciendo generacionalmente la baja calidad del sistema educativo nacional (Cristián Cox, en La Tercera del domingo 11 de abril de 2010).

16 www.futurolaboral.cl 
pesos mensuales, mientras que los jóvenes con padres que tienen educación superior obtendrían 1.206 .000 pesos mensuales. Por otro lado, la brecha de salarios se va reduciendo al considerar sólo a los que estudian o egresaron desde IP hasta no ser significativa para los salarios promedios de los egresados de CFT. Esta diferencia en los salarios en todo tipo de institución de educación superior indica que los jóvenes primera generación optan por carreras con menor retorno respecto de las elegidas por los jóvenes herederos.

Si bien la encuesta utilizada para este trabajo no provee información respecto del desempeño de los jóvenes durante la educación terciaria, igual es posible aproximarse a esta dimensión analizando la permanencia que se produce en este tipo de estudios ${ }^{17}$.

Los datos informan que del total de jóvenes primera generación, un 20,4\% desertó, en comparación al 7,4\% del grupo de jóvenes herederos. Esto significa que accedieron a estudios de educación superior y que en algún momento los abandonaron, y que hasta el momento de realizada la encuesta no han vuelto a retomarlos. Otra forma de entender este fenómeno es diciendo que del total de jóvenes que desertaron, un $83 \%$ de ellos son estudiantes primera generación.

El principal motivo para tomar la decisión de dejar los estudios superiores, declarado por ellos en la encuesta, son los problemas económicos. El 52\% de los jóvenes primera generación atribuye a esa causa el hecho de abandonar este tipo de educación. La mayoría de ellos deserta desde institutos profesionales (46\%) y universidades (39\%).

Como podemos concluir de este apartado, los jóvenes primera generación en educación superior si bien usan estrategias para acceder a este tipo de educación, de una u otra forma son frágiles, donde el factor económico finalmente termina pesando mucho al momento de tomar una decisión respecto a continuar estudiando.

17 Respecto a esto es importante recordar que la encuesta fue aplicada a los cuatro años de haber egresado de la educación media, razón por la cual es imposible rastrear fielmente el tipo de egreso de este tipo de educación. 


\section{Primera generación en educación superior, equidad y política educativa}

La descripción realizada respecto a las características de los jóvenes que en sus familias son los que por primera vez acceden a la educación superior, sin duda, da cuenta del estado de la equidad de todo nuestro sistema educativo. Esta situación se torna más interesante aún cuando esta descripción se sustenta en un enfoque de trayectorias, donde es posible identificar no sólo puntos de inicio y de llegada, sino también indagar sobre expectativas y estrategias que los sujetos ponen en juego para lograr sus objetivos, mostrando con ello, y en todo su potencial, la diversidad de recorridos que se encuentra presente hoy en nuestra educación.

A partir de esta perspectiva es posible observar la equidad en educación no sólo desde múltiples variables, sino también en una lógica temporal. Lo que hoy explica el posicionamiento que tienen los jóvenes primera generación en la educación superior es posible rastrearlo en diversos elementos que, de alguna forma, se han presentado e influido en las trayectorias que estos sujetos realizaron hasta ahora.

Considerando ello, es posible concluir que el origen de los jóvenes no explica por sí mismo las trayectorias que éstos terminan efectuando. En situaciones de precariedad socioeconómica similar, algunos jóvenes terminan su educación al finalizar la enseñanza media; en cambio otros logran acceder a la educación superior. Al parecer, la composición familiar (presencia de ambos padres y la presencia de hermanos) y el capital cultural de la familia (años de escolaridad de los padres y número de libros por hogar) terminan abriendo posibilidades para que los jóvenes puedan ingresar a la educación terciaria.

Un elemento que interpela de manera más directa a la política educativa es la influencia que tiene el mundo escolar en las trayectorias educativas postsecundarias, identificada por el estudio. Haber asistido a educación preescolar y tenido relativamente buen desempeño escolar dan como resultado la obtención de un puntaje más alto en la PSU, que claramente abre la posibilidad no sólo de entrar a la enseñanza 
superior, sino también de poder optar más libremente por carreras de mayor valoración social y retribución económica. En este sentido, seguir promoviendo una ampliación en la cobertura de la educación preescolar y mejorar la calidad de la enseñanza en los segmentos más vulnerables de la población son medidas que requieren seguir profundizándose desde esta perspectiva para que estos jóvenes puedan optar en igualdad de condiciones a continuar estudios superiores.

Específicamente, y como medida complementaria de las anteriores, es importante fortalecer las instancias de apoyo al tránsito entre la educación media y la educación superior. Como lo demuestra también este estudio, los jóvenes están accediendo a la enseñanza postsecundaria sin mucha claridad de lo que ésta significa (nuestros datos evidencian la desorientación existente respecto a qué estudiar y dónde, por ejemplo). Muchas veces ni sus familias ni sus más cercanos tienen un conocimiento claro de este nivel educativo. Como lo señalan otros estudios (Lagos y Palacios, 2008; y Rodríguez, Bahamondes y Becerra, 2008), tampoco sus colegios han servido lo suficiente como espacios de información y orientación a este respecto. Fortalecer la orientación vocacional se presenta como clave si se quiere mejorar el tránsito entre ambos niveles educativos.

El informe de la Organización para la Cooperación y el Desarrollo Económicos, OCDE, sobre educación superior dice: "Es necesario que los estudiantes tengan acceso a información completa y clara para poder elegir adecuadamente las instituciones, cursos y carreras. Además, necesitan explicación respecto a los programas académicos ofrecidos y a su calidad, costos, apoyo financiero disponible y posibilidad de completar sus estudios, conocer las perspectivas laborales del mercado y de desarrollar una carrera" (OCDE, 2009, p. 71).

Si bien es posible pensar que con el mejoramiento de los elementos antes expuestos sería factible abordar las principales dificultades que presentan los jóvenes que son primera generación en su inserción en la educación superior, es claro que éstas se mueven en una temporalidad de mediano o largo plazo. Desde el corto plazo, se puede mencionar lo que ya muchas instituciones han implementado en 
los últimos años, nos referimos a diagnósticos y programas remediales y/o de nivelación que han pretendido fortalecer las competencias de estudiantes menos aventajados o con más dificultades en su desempeño académico en sus primeros años de estudio. Las evidencias señalan ${ }^{18}$ que es importante que las instituciones monitoreen estas experiencias y velen por sus resultados, promoviendo una mayor responsabilidad respecto del logro académico de sus alumnos. Para ello se requiere modificar la idea de equidad que durante años ha reinado en la educación superior, que se enfoca más bien en el acceso a dicha enseñanza y no en la calidad de los procesos y resultados que genera. Evaluar los egresos y la deserción son dimensiones que es preciso ir posicionando cada vez más desde una perspectiva institucional y no sólo referida a las capacidades y desempeños de los propios estudiantes. El éxito o fracaso académico no dependen sólo de lo que los estudiantes hacen o dejar de hacer, el tipo de actuación institucional, al respecto, es igualmente importante.

La experiencia que algunas universidades han desarrollado en el formato de los llamados "propedéuticos"19 puede dar pistas interesantes no sólo acerca del tipo de acompañamiento necesario de entregar a los estudiantes con más rezago o dificultades educativas, sino también sobre los sistemas mismos de ingreso a la educación superior. Ciertas evidencias muestran que sobre la base de "cursos propedéuticos" algunas universidades han logrado, luego de dos años, que sus estudiantes más rezagados (aquellos que no ingresaron vía PSU) puedan nivelar sus competencias y habilidades respecto a aquellos que ingresaron a través de la postulación tradicional, vía PSU. Al parecer las instituciones de educación superior si se enfocan seriamente en este aspecto pueden compensar el handicap en desempeño que el contexto de origen de los estudiantes y las trayectorias escolares han ido generando durante los años anteriores.

18 Ver informe de la Educación Superior en Chile de la OCDE del año 2009.

19 Para más información ver las experiencias de la Universidad de Santiago de Chile (USACH), Universidad Católica Cardenal Silva Henríquez (UCSH) y Universidad Alberto Hurtado (UAH). 
Si bien es cierto que el hecho de concebir estrategias de apoyo para mejorar el desempeño de los estudiantes en la universidad es una clara señal de ir responsabilizándose por la actuación de éstos, el principal motivo para desertar de la educación superior, que los propios estudiantes mencionan, se relaciona con problemas económicos. Aunque los créditos y becas para la educación superior han ido progresivamente aumentando, es importante revisar los mecanismos y criterios de asignación de ellos, ya que no siempre aquellos que más lo necesitan pueden optar a dichos beneficios (OCDE 2009, p. 124).

Para finalizar, vemos en este tipo de perspectiva analítica que sitúa el enfoque de trayectorias un gran aporte para comprender mejor los procesos educativos y sus implicancias sociales, lo que sin duda es un insumo relevante para hacer una política más pertinente respecto de las nuevas dinámicas socioeducativas que se observan en el Chile de hoy. Necesitamos una institucionalidad que ponga en el centro de su gestión la complejidad del sujeto educativo, reconociendo su diversidad y dinamismo, aireando de esta forma el enfoque compartimentado y por "niveles" que los programas de mejoramiento de la educación han debido instalar para dar vida a esta política educativa en los últimos años. Esto es importante, ya que esta mirada, por muy técnica y experta que sea, no puede observar fenómenos que se dan en los tránsitos que los sujetos realizan entre niveles de enseñanza distintos, y que muchas veces pasan a ser centrales para entender las dinámicas que en ellos se producen.

\section{Referencias bibliográficas}

Armanet, Pilar. (2005) Formación Universitaria para el siglo XXI. En Foco. n. ${ }^{\circ}$ 44. Expansiva.

Baeza, Jorge (2007) La construcción de trayectorias en sociedades menos reguladas: desafíos al trabajo de Orientación Escolar en Educación Secundaria. En Foro Educacional. n. ${ }^{\circ}$ 12. Universidad Católica Silva Henríquez.

Bourdieu, Pierre (1980) Le sens pratique. Paris: Les Éditions de Minuit.

Bourdieu, Pierre (1988) Cosas dichas. Buenos Aires, Argentina: Editorial Gedisa S.A. 
74 CARACTERIZACIÓN DE JÓVENES PRIMERA GENERACIÓN EN EDUCACIÓN SUPERIOR - Jorge Castillo, Gustavo Cabezas

Bourdieu, Pierre (1991) El sentido práctico. Madrid, España: Ed. Taurus.

Bourdieu, Pierre (1994) Raisons pratiques sur la théorie de l'action. Paris: Éditions du Seuil.

Bourdieu, Pierre y Passeron, J-C. (2003) "Los Herederos. Los estudiantes y la cultura. Argentina". Argentina: Siglo XXI Editores.

Castillo, Jorge (2007) Demandas juveniles a la educación: la necesidad de fortalecer las trayectorias educativas en el sistema escolar. Revista Observatorio de Juventud. n. ${ }^{\circ}$ 16. Santiago.

Castillo, Jorge (2008) La equidad educativa en Chile: un análisis actual al sistema escolar y las políticas educativas. En Foro Educacional n. ${ }^{\circ} 14$. Universidad Católica Silva Henríquez.

Centro de Políticas Comparadas de Educación (2009) Primera generación en la universidad. Hijos de padres sin estudios superiores y su ingreso a la educación superior en Chile. Informe preliminar. UDP.

Charlot, Bernard (1997) Rapport au Savoir. Eléments pour une théorie. Paris: Ed. Anthropos.

Concha, Claudia (2009) Sujetos Rurales que por primera generación acceden a la universidad. Revista Calidad de la Educación n. ${ }^{\circ}$ 30. Consejo Nacional de Educación.

Contreras, Dante; Herrera, Rodrigo y Leyton, Gonzalo (2007) Impacto de la educación preescolar sobre el logro educacional. Evidencia para Chile.

Dávila, Óscar y Ghiardo, Felipe (2005) Trayectorias, transiciones y condiciones juveniles en Chile. Revista Nueva Sociedad. № 200. pp. 114-126.

Dávila, Óscar, Ghiardo, Felipe, Medrano, Carlos. (2005) Los Desheredados: Trayectorias de vida y nuevas condiciones juveniles. Santiago: Editorial CIDPA.

De Queiroz, J.M. et Ziolkovski M. (1997) Linteractionnisme symbolique. Paris : Presses Universitaires Rennes.

Dubet, François y Martuccelli, Danilo. (1998) En la escuela. Sociología de la experiencia escolar. Buenos Aires: Editorial Losada.

García Huidobro, Juan Eduardo (Editor) (2005) Políticas Educativas y Equidad. Reflexiones del Seminario Internacional. Santiago de Chile. Universidad Alberto Hurtado.

García Huidobro, Juan Eduardo y Bellei, Cristian (2003) Desigualdad Educativa en Chile. Santiago de Chile. Universidad Alberto Hurtado. Escuela de Educación. 
Ghiardo, Felipe y Dávila, Óscar (2005a) Curso y Discursos escolares en las trayectorias juveniles. Revista Última Década. n. ${ }^{\circ} 23$ CIDPA. pp. 33-76.

Gluz, Nora (2006) La construcción socioeducativa del becario: La productividad simbólica de las políticas sociales en la educación media. Buenos Aires, Argentina: Ed. Instituto Internacional de Planeamiento de la Educación, IIPE-UNESCO.

Goicovic, Igor. (2002) Educación y cultura juvenil secundaria. Educación, deserción escolar e integración laboral juvenil. En Última Década n. ${ }^{\circ} 16$ pp. 11-52.

Guichard, J. (1993) L'école et les représentations d'avenir des adolescents. Paris: Ed. PUF.

Instituto Nacional de la Juventud (2002) Evaluación de la reforma en cuanto a la permanencia de los jóvenes trabajadores en el sistema escolar. Santiago de Chile. INJUV.

Lagos, Felipe y Palacios, Fernanda (2008) Orientación vocacional y profesional en colegios de bajo nivel socioeconómico: percepciones de orientadores y estudiantes. Revista Calidad en la Educación n. ${ }^{\circ} 28$. Consejo Nacional de Educación.

La Tercera (2010) Docentes titulados llegan a su máximo histórico: más de diez mil en 2008. 11/04/2010 http://latercera.com/contenido/657_250670_9. shtml

López, Néstor (2005) Equidad educativa y desigualdad social. Desafíos de la educación en el nuevo escenario latinoamericano. IIPE-UNESCO.

Meneses, Francisco; Rolando, Rodrigo; Valenzuela Manuel y Vega, María Antonieta (2010) Ingreso a la Educación Superior: la experiencia de la cohorte de egreso 2005. Sistema Nacional de Información de la Educación Superior (SIES) y División de Educación Superior del MINEDUC.

Microdatos. Encuesta de seguimiento a egresados. Educación media técnicoprofesional. (2009). Santiago.

Mideplan (2006) Encuesta de Caracterización Socioeconómica Nacional. Santiago.

Mineduc (1998) Evaluación del marco institucional de la educación media técnicoprofesional. Santiago.

Montes, Nancy y Sendón, María Alejandra (2006) Trayectorias educativas de estudiantes del nivel medio. Argentina a comienzos del siglo XXI. Revista Mexicana de Investigación Educativa. Año/vol. 11, n. ${ }^{\circ}$ 029, México, pp. 381-402. 
76 CARACTERIZACIÓN DE JÓVENES PRIMERA GENERACIÓN EN EDUCACIÓN SUPERIOR - Jorge Castillo, Gustavo Cabezas

Navarro, L (2002) "Chile: Equidad social y educación en los años '90". IIPE - UNESCO.

OECD (2004) Revisión de Políticas Nacionales de Educación. El caso de Chile. OCDE. Santiago.

OCDE (2009) La Educación Superior en Chile, Revisión de Politicas Nacionales de Educación.

PIIE (2003) Estudio diagnóstico de la orientación en Chile. Santiago.

Programa de las Naciones Unidas para el Desarrollo, PNUD (2005) Expansión de la educación superior en Chile. Temas de Desarrollo Humano Sustentable. n. ${ }^{\circ}$ 10. Santiago.

Rodríguez, Bibiana; Bahamondes, Andrea y Becerra, Teresa (2008) Sistematización de ajustes al sistema educacional y a la institución escolar a la luz de la apropiación de nuevos enfoques de la orientación. Foro educacional. n. ${ }^{\circ}$ 14. Universidad Católica Silva Henríquez.

Sanhueza, Claudia. (2009) Tamaño de familia y orden de nacimiento en Chile: usando gemelos como experimento natural. Revista de Análisis Económico. Vol. 24. n. ${ }^{\circ}$ 2. pp. 133-152.

SITEAL (2006) Nuevas dinámicas familiares y su impacto en la escolarización de los adolescentes.

UAH-CIDE (2006) VI Encuesta Nacional de Actores del Sistema Educativo. Santiago.

Weinstein, José (2001) Joven y alumno. Desafíos de la enseñanza media. Última Década. n. ${ }^{\circ}$ 15. pp. 99-119.

Recibido: 16/04/2010

Aceptado: 25/05/2010 